\title{
Spectrum of Cirrhosis of Liver in Eastern Madhya Pradesh, India
}

\author{
Rahul Trimukhe ${ }^{1}$, Rahul Rai ${ }^{1}$ \\ ${ }^{I}$ (Department Of Medicine, NSCB Medical College and Hospital, Jabalpur, India)
}

\begin{abstract}
:
Background: - In India, etiology of cirrhosis varies with geographical areas.

Aim: - To evaluate etiological and clinical spectrum of cirrhosis in eastern Madhya Pradesh state of India.

Methods: - The study includes 125 cirrhotic patients (aged >14yrs) diagnosed by clinical, biochemical and radiological parameters admitted in NSCB medical college, Jabalpur between 2009 and 2011. All patients underwent routine lab investigations, and tests for hepatitis $B$ and hepatitis $C$ virus. Tests for Autoimmune, Wilson's disease and Haemochromatosis were also done. Patients were treated and outcome was noted. Results: - Mean age was $52 \pm 10$ Years, male constituted $71.2 \%$ cases. Alcoholism was seen in $36.8 \%$, HBV in $36.8 \%$ and $\mathrm{HCV}$ in $3.2 \%$ cases. Wilson's disease, cardiac cirrhosis was seen in $1(0.8 \%)$ patient each. No etiology could be found in $20.8 \%$ patients. Commonest presentation was ascites in (84.8\%), followed by jaundice (60\%), gastro-intestinal bleed (38.4\%), Hepatic encephalopathy (18.4\%), Hepatorenal syndrome (12\%) and Spontaneous Bacterial peritonitis (4.0\%). Maximum patients were in Child's Pugh B class (49\%). Main cause of mortality was HE and HRS; maximum deaths occurred in Child's C.

Conclusion: - Alcohol and HBV were major etiologies. Most common presentation was ascites and jaundice and maximum mortality was seen in Child's $C$.
\end{abstract}

Keywords : Cirrhosis, complications, etiology, India, outcome.

\section{Introduction}

In developed countries, alcohol and HCV are main causes of liver cirrhosis [1]. There have been no reports on epidemiology \& clinical presentation of cirrhosis of liver in eastern Madhya Pradesh. So we undertook this study to review spectrum of liver cirrhosis seen at N.S.C.B. Medical College Hospital Jabalpur in Madhya Pradesh. This study was done to find out various etiological factors causing cirrhosis of liver and to determine clinical spectrum and various complications and related mortality of cirrhosis.

\section{Methods}

This retrospective study included 125 patients of liver cirrhosis in Department of Medicine NSCB MCH, Jabalpur during September 2009 to December 2010. Only patients aged 14 and above were included. Patients were either admitted or seen as outpatients at Medical College Jabalpur. The following parameters were studied in all patients: age, sex, BMI, presenting symptoms, details of alcohol consumption, history of diabetes mellitus, physical signs at first presentation to our unit, liver profile (albumin, globulin, bilirubin, ALT, AST, alkaline phosphatase), hematological profile (hemoglobin, total and differential cell counts, Platelets), prothrombin time, renal profile (blood urea and serum creatinine)and blood sugar level. Hepatitis B surface antigen (HBsAg), Anti- HCV antibodies by radioimmunoassay and HIV ELISA were also done. All patients evaluated on ultrasonography of abdomen of frequency of $3-5 \mathrm{MHz}$ for: maximum vertical span of the liver, nodularity and presence of ascites. In patients with ascites, ascitic fluid routine, microscopy and culture/sensitivity was done

Other parameters were studied included serum iron, total iron binding capacity, serum ceruloplasmin, blood and urine copper levels, antinuclear factor (ANA), antimitochondrial antibody (AMA), antismooth muscle antibody (ASMA) wherever needed. $\mathrm{HBeAg}$, Anti-HBeAg, IgG \& IgM Anti- HBcAg were tested in HBsAgpositive and HCV RNA in Anti-HCV positive patients. HBV DNA was done wherever feasible. Radiological investigations such as Doppler of hepatoportal circulation, CT abdomen, MRI of abdomen were done whenever needed. UGI endoscopy and liver biopsy were be done when ever needed. All the symptoms, complications and outcomes were noted.

1. Etiology of cirrhosis of liver was considered as per following criteria.

1.1 Alcohol associated cirrhosis was occurring in patients with significant alcohol consumption. Latter was defined as almost daily intake of more than $40-80 \mathrm{gms}$ of alcohol in males and $20-40 \mathrm{gms}$ in females for period longer than 10 years $[2,3]$.

1.2 Hepatitis B associated cirrhosis was that occurring in patients with positive HBsAg.

1.3 Hepatitis $\mathrm{C}$ associated cirrhosis was that occurring in patients with positive anti $\mathrm{HCV}$ antibodies and detectable HCV RNA. 
1.4 Idiopathic cirrhosis included those with negative HBsAg, anti HCV antibodies and absence of history of significant alcohol consumption or other secondary causes.

1.5 Secondary causes included Wilson's disease, Hemochromatosis, autoimmune liver disease, primary biliary cirrhosis, non-alcoholic fatty liver disease, cardiac cirrhosis.

2. Physical signs and complications.

2.1 Spontaneous bacterial peritonitis was diagnosed on positive culture of the peritoneal fluid, in patient of ascites and absence of any obvious intra-abdominal source [4].

2.2 Splenomegaly was diagnosed on ultrasonography.

2.3 Upper gastrointestinal bleeding (UGI Bleed) was considered when patient had history of melena or hemetemesis and evidence of bleed on UGI endoscopy.

2.4 Hepatorenal Syndrome was considered when serum creatinine or creatinine clearance is less than $40 \mathrm{ml} / \mathrm{min}$ with absence of any intrinsic renal impairment or any nephrotoxic drugs intake.

2.5 Hepatic encephalopathy was defined as when symptoms and signs of liver cell failure with psycho neurological impairment.

2.6 Thrombocytopenia was defined as platelets less than 150,000/cu mm.

All the patients were categorized as per Child Turcotte Pugh Criteria (Table 1). The probability level of $<0.05$ was set for statistical significance. SPSS 10 (SPSS Inc, Chicago, IL) was used for statistical computations.

\section{Results}

Chronic alcoholism associated cirrhosis was seen in 46 patients (all were males) followed by HBV related in 46 patients (one had HBV HIV co-infection; females constituted 66\% of HBV infection) and HCV related in 4 cases. One each patient of Wilson's disease and cardiac cirrhosis was noted. No etiology could be found in 26 patients. Mean age was $52 \pm 10$ years, male constituted $71.2 \%$ cases out of 125 patients.

Commonest presentation was ascites in $(84.8 \%)$, followed by jaundice $(60 \%)$, Splenomegaly $(57.6 \%)$, UGI bleed (38.4\%), thrombocytopenia (20\%), Hepatic encephalopathy (18.4\%), Hepatorenal syndrome (12\%) and Spontaneous Bacterial peritonitis (4.0\%). Main cause of mortality was hepatic encephalopathy and hepatorenal syndrome. Maximum percentage mortality was seen in alcoholic cirrhosis followed by cryptogenic cirrhosis then HBV related cirrhosis (Table-2).

\section{Discussion}

In this study alcohol and HBV related cirrhosis were commonest etiologies followed by cryptogenic, $\mathrm{HCV}$ related, Wilson's disease and cardiac cirrhosis. Worldwide alcohol and HCV related cirrhosis are common. In a study at Goteborg University in Sweden, incidence of alcoholic cirrhosis was $48 \%$, Hepatitis C related cirrhosis in 10\%, Hepatitis B related cirrhosis in 3\%, NAFLD in 15\%, and primary biliary cirrhosis in 5\% and others causes constituted 7\% [5] Thus in developing countries incidence of cirrhosis due to hepatitis B is higher than cirrhosis due to hepatitis $\mathrm{C}$ in contrast to western population, as seen in our study. Even in India different geographical area have different causes of cirrhosis. In eastern India HBV related cirrhosis was the commonest cause of cirrhosis.

All alcoholic cirrhotic patients were males as compared to male to female ratio in western population which ranges from 2 to 10:1. In HBV related cirrhosis, there was female preponderance and male to female ratio was 1:1.8. Mean age of our cirrhotic patients was 52 years, which was similar to that noted by Kudva in Malaysia [6]. HBV related cirrhosis occurred in younger age (46years) compared with alcoholic related type (54years). Studies elsewhere shown that the age of presentation of symptoms in HBV is about 42 years whereas for alcoholic cirrhosis it is 50 years.

Almost $80 \%$ of our patients were presented with decompensation, particularly ascites. The frequency of with which patients presented with jaundice, splenomegaly and UGI bleed were similar to other studies. In the Goteborg University ascites was most common complication in $61 \%$ followed by UGI bleeding in 55\%, hepatocellular carcinoma in $11 \%$ and portal hypertension in $33 \%$ cases [5]. In our study, most patients were in Child Turcotte Pugh class B category followed by class C. Maximum mortality was noted in class C, who were presented with hepatic encephalopathy (80\%) and hepatorenal syndrome (60\%) followed by jaundice (58\%) and UGI bleed (40\%). 


\section{Tables}

Table 1: Child Turcotte Pugh Criteria for categorizing disease severity in cirrhotic patients.

\begin{tabular}{|ll|c|c|c|}
\hline \multicolumn{2}{|c|}{ Parameter } & 1 Point & 2 Points & 3 Points \\
\hline Serum Bilirubin & $\mathrm{mol} / \mathrm{L}$ & 34 & $34-51$ & $>51$ \\
& $\mathrm{Mg} / \mathrm{dL}$ & 2.0 & $2.0-3.0$ & $>3.0$ \\
\hline \multirow{2}{*}{ Serum Albumin } & $\mathrm{g} / \mathrm{L}$ & $>35$ & $30-35$ & $<30$ \\
& $\mathrm{~g} / \mathrm{dL}$ & $>3.5$ & $3.0-3.5$ & $<3.0$ \\
\hline Prothrombin time & $\mathrm{sec}$ prolonged & $0-4$ & $4-6$ & $>6$ \\
$\mathrm{INR}$ & & $<1.7$ & $1.7-2.3$ & $>2.3$ \\
\hline Ascites & None & Easily & Poorly \\
& & & controlled & controlled \\
\hline \multicolumn{2}{|c|}{ Hepatic Encephalopathy } & None & Minimal & Advanced \\
\hline
\end{tabular}

Child Turcotte Pugh class A (compensated cirrhosis): score $<7$; Child Pugh class B (significant functional compromise): score 8-10; Child Pugh class C (decompensated cirrhosis): score $>11$

Table 2: Presenting features and complications in patients with liver cirrhosis

\begin{tabular}{|l|l|l|l|l|l|}
\hline Presenting Feature & $\begin{array}{l}\text { Alcoholic } \\
\text { Cirrhosis }\end{array}$ & HBV Cirrhosis & Idiopathic & Others & All Patients \\
\hline Ascites & $89 \%$ & $87 \%$ & $81 \%$ & $57 \%$ & $84.8 \%$ \\
\hline Jaundice & $54 \%$ & $65 \%$ & $61 \%$ & $57 \%$ & $60 \%$ \\
\hline Splenomegaly & $70 \%$ & $54 \%$ & $50 \%$ & $29 \%$ & $57.6 \%$ \\
\hline UGI Bleed & $43 \%$ & $33 \%$ & $46 \%$ & $14 \%$ & $38.4 \%$ \\
\hline Thrombocytopenia & $22 \%$ & $13 \%$ & $34 \%$ & 0 & $20 \%$ \\
\hline Hepatic encephalopathy & $22 \%$ & $11 \%$ & $31 \%$ & 0 & $18.4 \%$ \\
\hline Hepatorenal syndrome & $17 \%$ & $4 \%$ & $23 \%$ & 0 & $12 \%$ \\
\hline $\begin{array}{l}\text { Spontaneous Bacterial } \\
\text { peritonitis }\end{array}$ & $6 \%$ & $2 \%$ & $4 \%$ & 0 & $4 \%$ \\
\hline
\end{tabular}

\section{Conclusion}

Alcohol and HBV infection were main causes for cirrhosis in this part of India; females outnumbered males in HBV infection. Most common presentations were ascites and jaundice. Most patients presented in Child Turcotte Pugh category B but maximum mortality was seen in class C. Public health measures to control alcohol abuse and management of chronic HBV infection can reduce the incidence of liver cirrhosis. The major limitation of study was a smaller sample size. The diagnosis of cirrhosis predominantly was a clinical and radiological, histology could not be done in any of presumed cirrhotics.

\section{References}

[1]. Nguyen GC, Segev DL, Thuluvath PJ. Nationwide increase in hospitalizations and hepatitis C among inpatients with cirrhosis and sequelae of portal hypertension. Clin Gastroenterology Hepatol 2007;5:1092-1099.

[2]. Fuchs CS, Stampfer MJ, Colditz GA, et al: Alcohol consumption and mortality among women. N Engl J Med 1995; 332:1245-50.

[3]. Becker U, Deis A, Sorensen TI, et al: Prediction of risk of liver disease by alcohol intake, sex, and age: A prospective population study. Hepatology 1996; 23:1025-9.

[4]. Runyon BA, Hoefs JC, Morgan TR: Ascitic fluid analysis in malignancy-related ascites. Hepatology 1988; 8:1104-9.

[5]. Gunnarsdóttir SA. Liver cirrhosis-epidemiological and Clinical Aspects. Inst of Medicine. Dept of Internal Medicine; 2008 May 19.

[6]. M V Kudva: Chronic liver disease in Kuala Lumpur, Malaysia: clinical study; Singapore Med J 1990; vol 31:368-373. 